\title{
Gender sorting among economists: Evidence from the NBER
}

\author{
Benjamin Davies*
}

May 20, 2022

\begin{abstract}
I compare the co-authorship patterns of male and female economists, using historical data on National Bureau of Economic Research working papers. Men tended to work in smaller teams than women, but co-authored more papers and so had more co-authors overall. Both men and women had more same-gender co-authors than we would expect if co-authorships were random. This was especially true for men in Macro/Finance.
\end{abstract}

JEL classification: A14; D85; J16

Keywords: co-authorship; gender; homophily; NBER; networks

${ }^{*}$ Department of Economics, Stanford University; bldavies@stanford.edu. I thank Akhila Kovvuri, Anirudh Sankar, Florencia Hnilo, Lukas Bolte, and an anonymous referee for comments. 


\section{Introduction}

The National Bureau of Economic Research (NBER) is a North American organization focused on facilitating and disseminating economic research. Its working paper series, launched in 1973, is the premier such series in economics (Lusher et al., 2021). This paper compares the co-authorship patterns of male and female authors of NBER working papers published before $2020{ }^{1}$

Men tended to work in smaller teams than women, but co-authored more papers and so had more co-authors overall. Both men and women had more same-gender co-authors than we would expect if co-authorships were random. This was especially true for men in Macro/Finance. My analysis complements that by Ductor et al. (2021) and Ductor and Prummer (2021), who find similar patterns for journal articles but do not analyze variation in gender sorting between subfields of economics. This similarity suggests that gender differences in co-authorship patterns are not artefacts of peer review.

These co-authorship patterns reveal one form of gender disparity among research economists. Other forms include female under-representation (Bayer and Rouse, 2016; Ginther and Kahn, 2004; Lundberg, 2018; Meade et al., 2021), unbalanced professional standards (Card et al., 2020; Hengel, 2017; Koffi, 2021; Sarsons, 2017; Sarsons et al., 2021), and differential treatment in seminars (Dupas et al., 2021). Gender sorting may restrict authors' access to human and social capital provided by collaborators, limiting authors' ability to conduct socially valuable research.

\section{Data construction and description}

My data cover all 27,059 unique NBER working papers published during the years 1973 through 2019. I derive these data from raw metadata published on the NBER's website. ${ }^{2}$ My derived data are available via the R package nberwp (Davies, 2022). ${ }^{3}$

The NBER allocates papers to "programs" corresponding to branches of economics. Table A.1 counts papers by program and decade. Overall, about a third of papers were associated with the largest three programs: Labor Studies, Economic Fluctuations and Growth, and Public Economics. Most papers are recent: more than half were published in 2007 or later.

The papers in my data had 13,636 authors, whom I disambiguate as follows. The raw metadata link authors to their papers, and their usernames on the NBER and Research Papers in Economics (RePEc) websites. I use these usernames to identify authors who published under multiple names. I then identify authors who (i) had similar names, and either (ii) shared a common co-author or (iii)

\footnotetext{
${ }^{1}$ I end my analysis before 2020 because the COVID-19 pandemic differentially affected men and women's abilities to publish new papers (Deryugina et al., 2021), which may influence subsequent co-authorship patterns.

${ }^{2}$ See https:/ / data.nber.org/nber-wp-logs/ and https://www2.nber.org/RePEc/nbr/ (accessed August 29, 2021).

${ }^{3}$ See https:/ / github.com/bldavies/nberwp/ (accessed January 12, 2022) for the code used to derive my data.
} 
Table 1: Number of authors by gender and source of gender information

\begin{tabular}{lrrrr}
\hline Gender & SSA & Facebook & Manual & Total \\
\hline Female & 2,627 & 131 & 626 & 3,384 \\
Male & 8,002 & 415 & 1,689 & 10,106 \\
Unknown & - & - & 146 & 146 \\
\hline Total & 10,629 & 546 & 2,461 & 13,636 \\
\hline
\end{tabular}

Notes: "SSA" = 1940-1995 Social Security Administration baby names. "Facebook" = Facebook names collected by Tang et al. (2011). Genders not determined via SSA or Facebook data are identified manually. "Unknown" genders arise only from manual identification failures.

published in the same program. ${ }^{4}$ Applying criteria (i)-(iii) yields a set of disambiguated authors. I check this set for authors with identical names, resolving false splits manually. I supplement these steps by manually resolving errors found while cleaning and analyzing my data.

I determine authors' genders as follows. First, I match authors' forenames to baby name data from the United States (US) Social Security Administration. These data report the share of babies with each forename born in the US between 1940 and 1995 who were girls. ${ }^{5}$ I classify an author as female if this share was larger than $95 \%$ and as male if it was smaller than $5 \% .{ }^{6}$ I then match unmatched forenames to Tang et al.'s (2011) data on Facebook users' forenames and self-reported genders, and apply the 95\% and 5\% classification rules again using those data. Finally, I manually identify the gender of authors with genders undetermined by the baby name or Facebook data. Manual identification involves searching for gendered pronouns in online biographies, news articles, and professional and social media profiles. If such materials are unavailable or conflict then I classify the author's gender as unknown. ${ }^{7}$

Table 1 counts authors in my data by gender and source of gender information. I determine $78 \%$ of authors' genders from baby names and a further $4 \%$ using Facebook data. ${ }^{8}$ About a quarter

\footnotetext{
"I define "similar" to mean "share a Cosine edit distance less than 0.05 ," along with some other edit distance thresholds specific to criteria (ii) and (iii). I establish these thresholds by manually verifying that they deliver correct results. Criteria (ii) and (iii) are heuristics: (ii) assumes authors tend to write papers with the same set of co-authors, while (iii) assumes authors with similar names and academic interests are likely the same person.

${ }^{5}$ I use 1940-1995 data because that period corresponds to (what I assume are) the birth years of authors who published NBER working papers between 1973 and 2019.

${ }^{6}$ This rule may mis-classify some authors due to different naming practices outside the US. For example, "Andrea" and "Simone" are typically female names in the US but male names in Italy. I check and correct such mis-classifications manually, using the genderize.io API to identify potentially mis-classified names as those given to less than $95 \%$ of men or women across all countries. The necessary corrections are few and do not change my results.

${ }^{7}$ This may reflect authors with non-binary genders.

${ }^{8}$ The share of genders determined from baby names was smaller in later publication years, reflecting increasing authorship of NBER working papers by researchers born outside the US.
} 
Table 2: Female representation across NBER research programs and decades

\begin{tabular}{|c|c|c|c|c|c|c|c|}
\hline \multirow[b]{2}{*}{ Subfield } & \multirow[b]{2}{*}{ Program } & \multicolumn{6}{|c|}{$\%$ unique authors who were female } \\
\hline & & 1970s & $1980 \mathrm{~s}$ & $1990 \mathrm{~s}$ & $2000 s$ & $2010 s$ & Overall \\
\hline \multirow[t]{7}{*}{ Macro/Finance } & International Trade and Investment & 0.0 & 12.9 & 14.0 & 21.2 & 22.4 & 20.4 \\
\hline & Corporate Finance & 0.0 & 0.0 & 9.8 & 19.3 & 19.0 & 19.0 \\
\hline & International Finance and Macroeconomics & 0.0 & 12.8 & 12.0 & 15.2 & 18.9 & 17.1 \\
\hline & Economic Fluctuations and Growth & 0.0 & 5.3 & 7.1 & 15.7 & 16.8 & 16.4 \\
\hline & Asset Pricing & - & - & 8.7 & 14.8 & 16.5 & 15.8 \\
\hline & Monetary Economics & 0.0 & 4.5 & 9.3 & 12.7 & 15.2 & 13.7 \\
\hline & Overall & 0.0 & 9.2 & 11.0 & 18.0 & 19.6 & 18.9 \\
\hline \multirow[t]{15}{*}{ Micro } & Children & 0.0 & 25.0 & 36.2 & 32.3 & 38.5 & 37.5 \\
\hline & Health Economics & 25.0 & 16.1 & 22.6 & 32.2 & 34.0 & 33.8 \\
\hline & Economics of Education & - & - & 33.3 & 28.1 & 34.2 & 33.4 \\
\hline & Health Care & - & - & 27.8 & 30.0 & 33.9 & 33.1 \\
\hline & Development Economics & - & - & - & - & 28.3 & 28.3 \\
\hline & Labor Studies & 9.4 & 16.1 & 25.1 & 24.9 & 28.5 & 27.6 \\
\hline & Economics of Aging & 0.0 & 5.9 & 20.3 & 23.6 & 28.0 & 26.9 \\
\hline & Public Economics & 3.6 & 7.2 & 14.3 & 20.3 & 25.4 & 23.4 \\
\hline & Law and Economics & 0.0 & 0.0 & 8.3 & 19.4 & 24.3 & 22.9 \\
\hline & Productivity, Innovation, and Entrepreneurship & 0.0 & 13.3 & 21.3 & 21.0 & 22.6 & 22.5 \\
\hline & Environment and Energy Economics & - & 0.0 & 4.8 & 20.6 & 22.3 & 22.3 \\
\hline & Development of the American Economy & 66.7 & 15.6 & 19.3 & 21.4 & 21.5 & 22.1 \\
\hline & Industrial Organization & - & - & 17.4 & 20.7 & 19.4 & 19.9 \\
\hline & Political Economics & - & - & - & 14.5 & 19.1 & 18.9 \\
\hline & Overall & 10.4 & 12.7 & 20.9 & 24.4 & 27.5 & 27.0 \\
\hline Overall & & 7.6 & 12.3 & 17.6 & 22.3 & 26.1 & 25.1 \\
\hline
\end{tabular}

Notes: Bottom row percentages include papers associated with Technical Working Papers program or with no associated programs. All percentages exclude authors with unknown gender. Zeroes are true; hyphens indicate program-decade pairs with no male or female authors.

of authors were women, both overall and for each source.

Table 2 shows how female representation grew during my period of study and how this growth varied by program. I allocate programs to the "Micro" or "Macro/Finance" subfield similarly to Chari and Goldsmith-Pinkham (2017). Women were more represented in Micro programs, especially in programs focused on children, health, and education. Women were least represented in the Monetary Economics and Asset Pricing programs. These patterns echo those in NBER conference submissions (Chari and Goldsmith-Pinkham, 2017) and journal articles (Meade et al., 2021). 


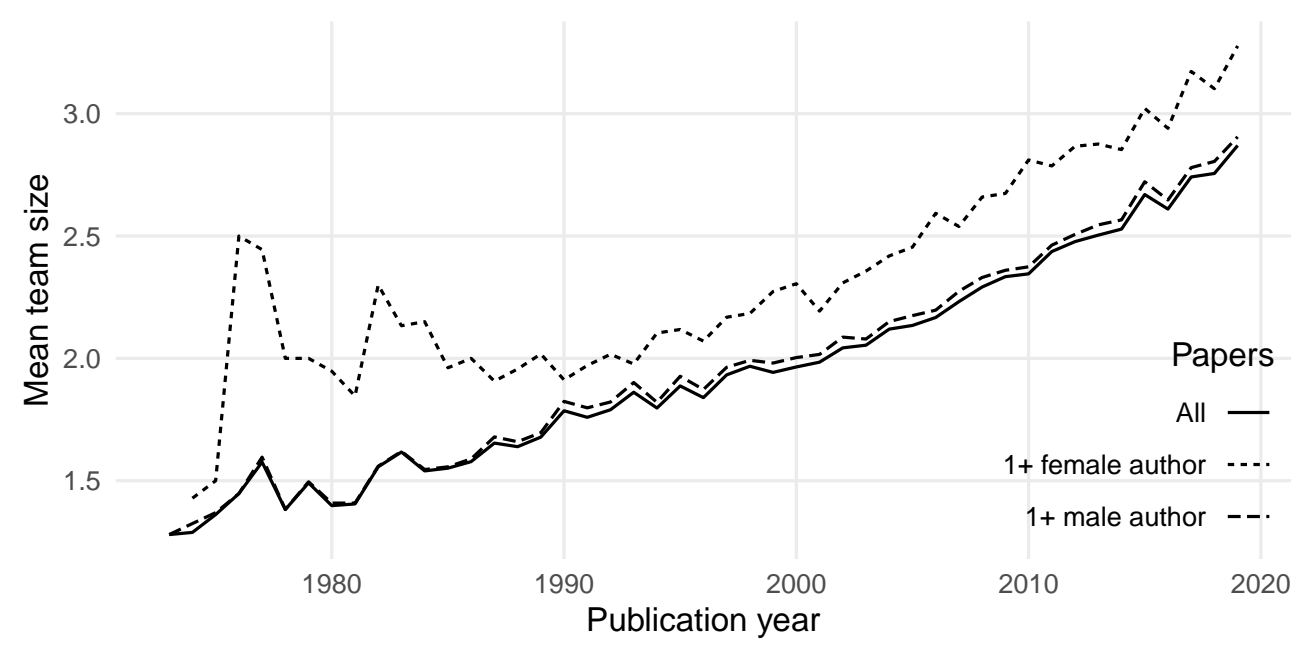

Figure 1: Rising team sizes on NBER working papers

Notes: Vertical axis measures mean number of authors on papers published in each year. " $1+$ female author" = papers with at least one female author. " $1+$ male author" = papers with at least one male author. Both series exceed "All" due to positive selection on team size. See Figure A.3 for confidence intervals around means.

\section{Co-authorship patterns}

Figure 1 shows that the mean co-authorship team size on NBER working papers grew from 1.28 in 1973 to 2.87 in 2019. Teams with at least one woman tended to be larger than teams with at least one man. This tendency remains if I exclude solo-authored papers, which became less frequent over time.

Figure A.1 shows that the rise in team sizes coincided with a shift in team compositions, mostly from all-male teams to mixed gender teams. The share of all-female teams was about $5 \%$ each year during my period of study. Figure A.2 shows that team composition did not covary significantly with whether papers appeared in peer-reviewed journals. Team composition may covary with citation accrual—see, e.g., Ductor and Prummer (2021), Koffi (2021), and Maddi and Gingras (2021) for conflicting evidence. However, such patterns are beyond the scope of this paper.

\subsection{The co-authorship network}

I define a time-varying co-authorship network $N_{t}$, in which nodes represent authors who published NBER working papers in years $(t-9)$ through $t$, and edges join authors who co-authored at least once during those years. ${ }^{9}$ This network tracks the birth and decay of collaborative relationships: deleting edges after many years of inactivity captures how the human and social capital embodied in those connections fade over time if not renewed.

\footnotetext{
${ }^{9}$ My results do not change qualitatively if I define $N_{t}$ using a shorter publication window.
} 


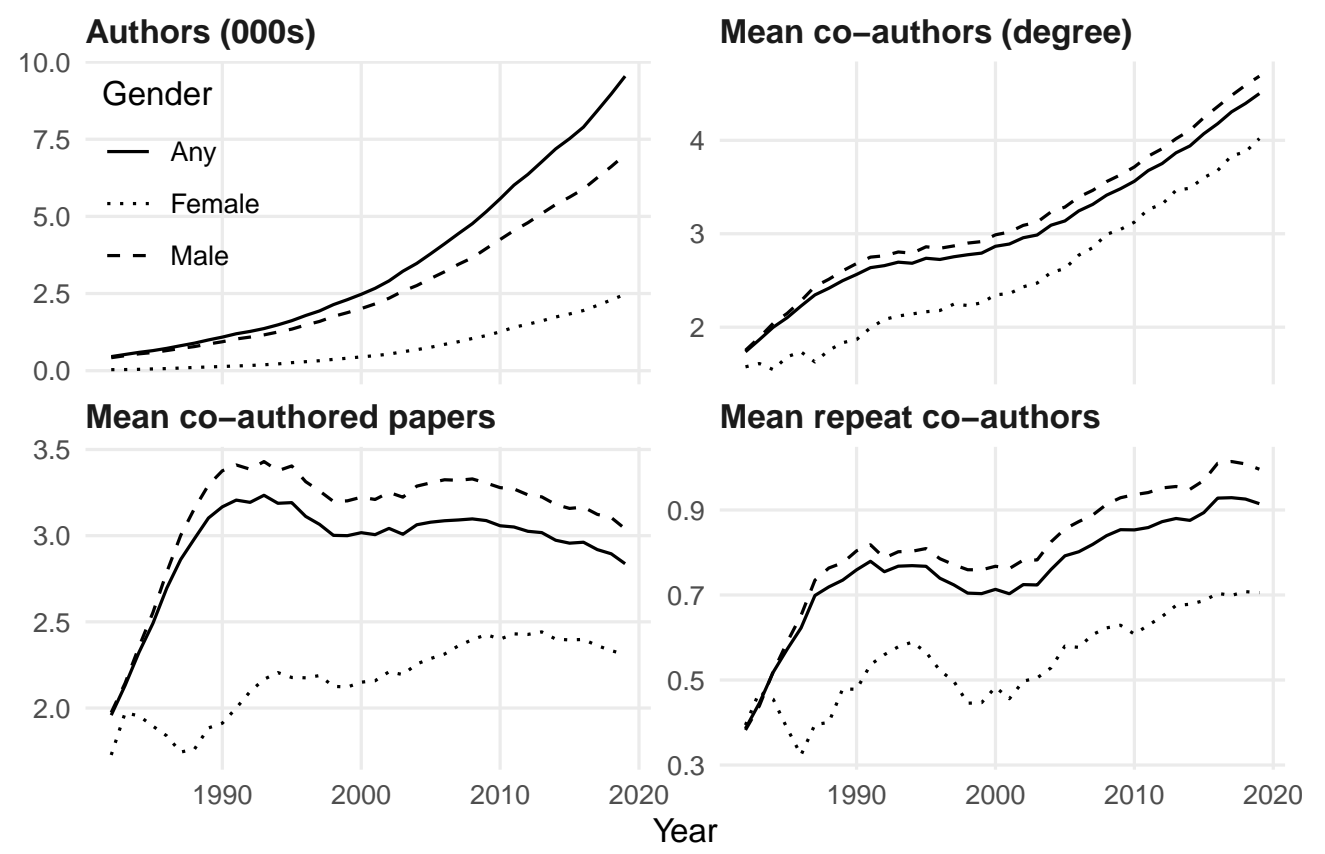

Figure 2: Trends among authors in the co-authorship network $N_{t}$

Notes: "Any" series include authors with unknown genders. "Co-authored papers" = unique papers co-authored with at least one other author during the years $(t-9)$ through $t$. "Repeat co-authors" = unique co-authors on more than one paper during the years $(t-9)$ through $t$. Means computed across all authors in $N_{t}$ with each gender. See Figure A.4 for confidence intervals around means.

Figure 2 shows that the number of authors in $N_{t}$ grew over time, as did the proportion of those authors who were women. The mean degree (i.e., co-author count) also grew, both overall and for each gender. Men tended to have more (new and repeat) co-authors and co-author more papers than women. ${ }^{10}$

\subsection{Gender sorting}

I measure the extent of gender sorting in the co-authorship network $N_{t}$ using Newman's (2003) "assortativity coefficient"

$$
r_{t}=\frac{\sum_{g \in G} x_{t g g}-\sum_{g \in G} y_{t g}^{2}}{1-\sum_{g \in G} y_{t g}^{2}},
$$

where $x_{t g g^{\prime}}$ is the share of edges joining authors of gender $g \in G=\{$ Female, Male $\}$ to authors of gender $g^{\prime} \in G$, and where

$$
y_{t g}=\sum_{g^{\prime} \in G} x_{t g g^{\prime}}
$$

\footnotetext{
${ }^{10}$ Men also tended to publish more solo-authored papers than women.
} 


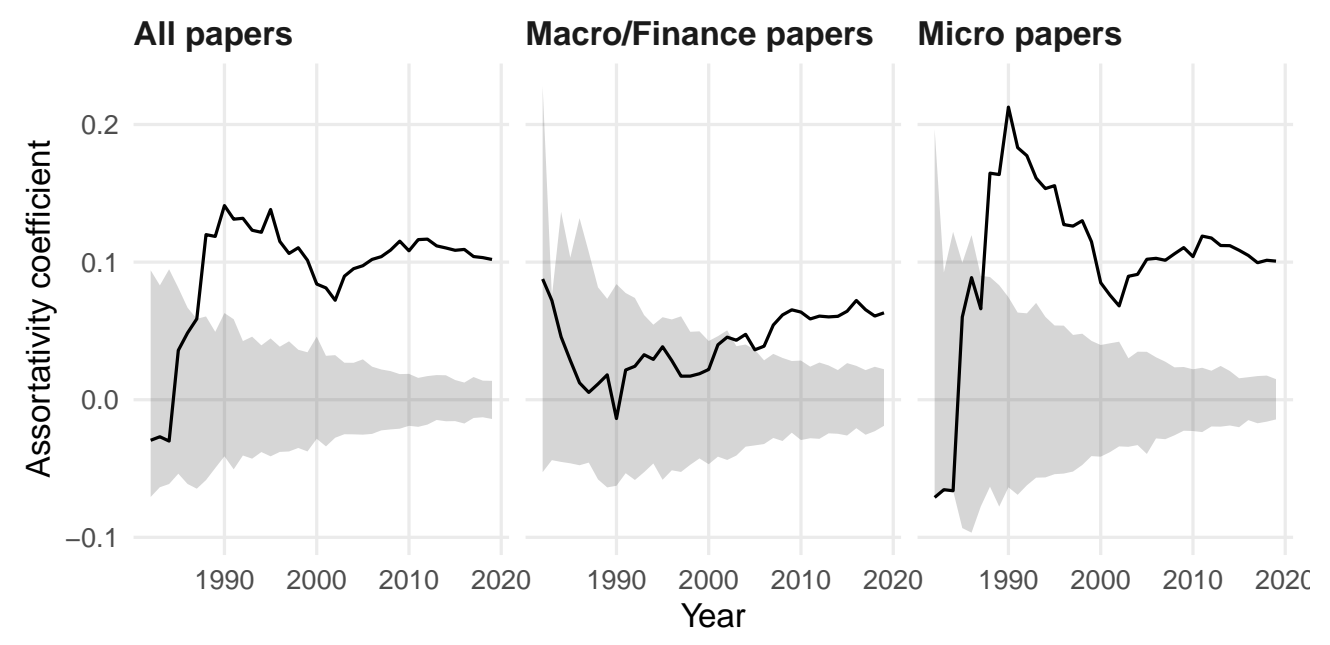

Figure 3: Gender sorting in the co-authorship network $N_{t}$

Notes: Shaded regions represent 95\% confidence intervals across 200 gender- and degree-preserving randomizations of $N_{t}$ and its subfield subnetworks. Coefficients exclude authors with unknown gender.

is the share of edges incident with an author of gender $g$. The coefficient $r_{t}$ varies between -1 and 1 , is larger when $N_{t}$ is more sorted (i.e., within-gender edges are more common than betweengender edges), and equals 0 when $N_{t}$ is unsorted (i.e., edges "look random"). It equals the Pearson correlation of the genders of pairs of authors who co-authored during years $(t-9)$ through $t$ (e.g., treating "male" as zero and "female" as one).

Figure 3 tracks the assortativity coefficient for the network $N_{t}$, and for the networks obtained after restricting to co-authorships of papers in the Micro and Macro/Finance subfields defined in Table 2. The figure also shows the $95 \%$ confidence interval for the assortativity coefficient of a random network among the authors in $N_{t}$, holding their genders and degrees constant. This interval summarizes the distribution of coefficients obtained by fixing authors' genders and propensities to co-author, but randomizing whom they co-authored with. ${ }^{11}$ Coefficients outside this interval indicate statistically significant sorting.

The network $N_{t}$ showed positive gender sorting from 1985 onwards. Such sorting was significantly greater than a gender- and degree-preserving random network from 1988 onwards, with the extent of sorting fluctuating around $r_{t}=0.1$. Thus, author genders have been weakly predictive of co-authors' genders since the late 1980s.

Gender sorting was strongest among authors of Micro papers. This could be due to betweengender variation. For example, there were relatively few women in Macro/Finance (see Table 2),

\footnotetext{
${ }^{11}$ The interval is centred at zero because random networks tend to be unsorted. It shrinks with $t$ because the number of authors in $N_{t}$ grew, so if co-authorships were random then positive sorting among some authors would have become more likely to be "cancelled out" by negative sorting among others.
} 


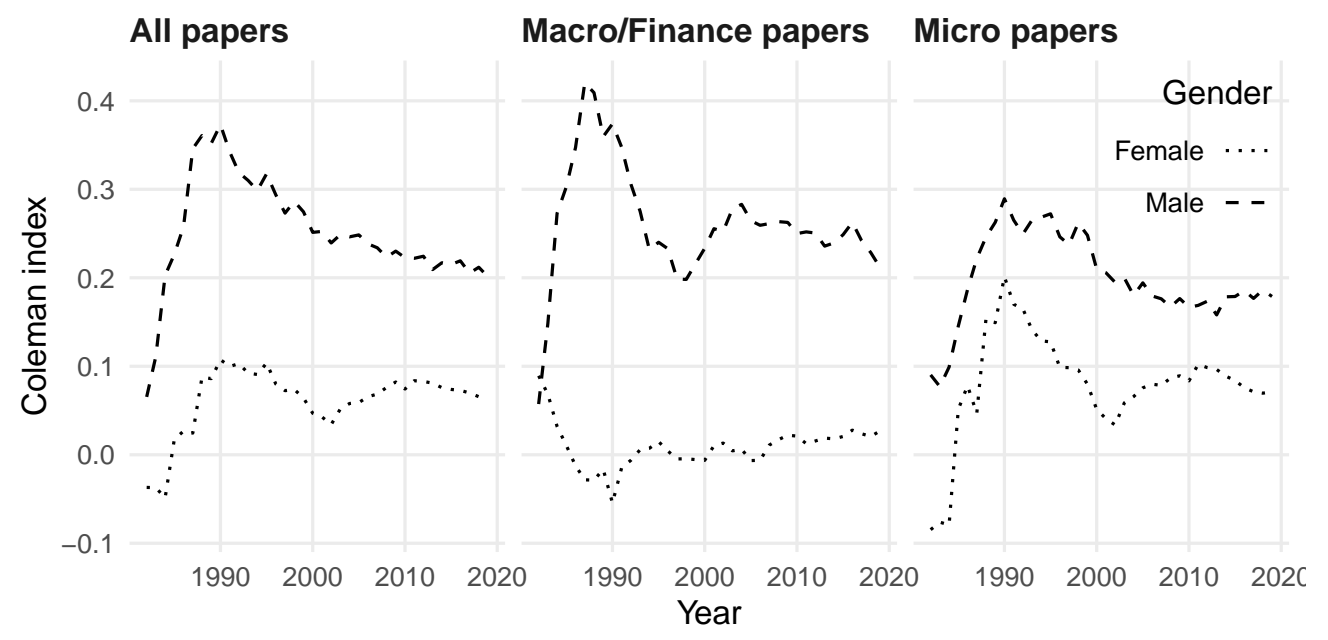

Figure 4: Coleman indices for men and women in $N_{t}$ and its subfield subnetworks

Note: Indices exclude authors with unknown gender.

who thus had relatively few choices of same-gender co-authors. I explore this possibility by computing Coleman's (1958) index

$$
C_{t g}=\frac{H_{t g}-w_{t g}}{1-w_{t g}}
$$

where $H_{t g}$ is the share of edges incident with gender $g$ authors that are within-gender, and $w_{t g}$ is the share of authors with gender $g$. The numerator of $C_{t g}$ captures the rate at which authors of gender $g$ co-authored with each other in excess of what we would expect if co-authorships were uniformly random. The denominator normalizes this excess by its maximum possible value to adjust for variation in shares $w_{t g}$. Thus $C_{t g}$ measures how much excess same-gender co-authorship among authors of gender $g$ there was relative to how much there could have been.

Figure 4 shows that men had larger contemporaneous Coleman indices than women. Men in Macro/Finance had the largest indices while women in that subfield had the smallest indices.

Figures 3 and 4 show temporal variation in gender sorting, such as a sharp rise during the late 1980s for Micro authors and male Macro/Finance authors. This variation may come from the NBER launching programs (e.g., Asset Pricing in 1991 and Children in 1996) with varying female representation. ${ }^{12}$ I leave exploring this hypothesis to future research.

\footnotetext{
${ }^{12}$ The raw metadata contain retroactive paper-program associations, so some programs have papers associated with them before they were launched.
} 


\section{Concluding remarks}

This paper documents recent and historical gender sorting among co-authors of NBER working papers. Both men and women had more same-gender co-authors than we would expect if coauthorships were random. This was especially true for men in Macro/Finance. Gender sorting may restrict authors' access to resources provided by collaborators, limiting authors' ability to conduct socially valuable research and advance their careers.

My analysis is silent on mechanisms driving gender sorting and on potential confounders. Female academic economists tend to begin their careers in lower-ranked departments than men, leading women to have less productive co-authors (Ghosh and Liu, 2020). Figure 2 suggests that such co-authors tend to be women. Thus, gender sorting could be driven by factors correlated with gender rather than by gender itself.

Ultimately, co-authorships are outcomes of search and matching processes. My analysis does not account for constraints on such processes. For example, the relative under-representation of women in Macro/Finance may prevent new authors in that subfield from finding female collaborators or advisors with similar interests, independently of their matching preferences. This may, in turn, restrict economists' academic opportunities, as well as dampen the profession's ability to attract talent and generate impactful research. Studying the severity and impact of constraints on co-author matching processes, and initiatives to relax those constraints, may improve the profession and its social impact.

\section{References}

Bayer, A. and Rouse, C. E. (2016). Diversity in the Economics Profession: A New Attack on an Old Problem. Journal of Economic Perspectives, 30(4):221-242.

Card, D., DellaVigna, S., Funk, P., and Iriberri, N. (2020). Are Referees and Editors in Economics Gender Neutral? Quarterly Journal of Economics, 135(1):269-327.

Chari, A. and Goldsmith-Pinkham, P. (2017). Gender Representation in Economics Across Topics and Time: Evidence from the NBER Summer Institute. NBER Working Paper w23953, National Bureau of Economic Research, Cambridge, MA.

Coleman, J. (1958). Relational Analysis: The Study of Social Organizations with Survey Methods. Human Organization, 17(4):28-36.

Davies, B. (2022). nberwp: NBER Working Papers. R package version 1.2.0.

Deryugina, T., Shurchkov, O., and Stearns, J. (2021). COVID-19 Disruptions Disproportionately Affect

Female Academics. AEA Papers and Proceedings, 111:164-168.

Ductor, L., Goyal, S., and Prummer, A. (2021). Gender and Collaboration. Review of Economics and Statistics, forthcoming.

Ductor, L. and Prummer, A. (2021). Gender Diversity in Teams. 
Dupas, P., Modestino, A. S., Niederle, M., Wolfers, J., and The Seminar Dynamics Collective (2021). Gender and the Dynamics of Economics Seminars. NBER Working Paper w28494, National Bureau of Economic Research, Cambridge, MA.

Ghosh, P. and Liu, Z. (2020). Coauthorship and the gender gap in top economics journal publications. Applied Economics Letters, 27(7):580-590.

Ginther, D. K. and Kahn, S. (2004). Women in Economics: Moving Up or Falling Off the Academic Career Ladder? Journal of Economic Perspectives, 18(3):193-214.

Hengel, E. (2017). Publishing while female. Cambridge Working Papers in Economics 1753, University of Cambridge.

Koffi, M. (2021). Innovative Ideas and Gender Inequality. CLEF Working Paper 35, University of Waterloo. Lundberg, S. (2018). Report: Committee on the Status of Women in the Economics Profession (CSWEP). AEA Papers and Proceedings, 108:704-721.

Lusher, L. R., Yang, W., and Carrell, S. E. (2021). Congestion on the Information Superhighway: Does Economics Have a Working Papers Problem? NBER Working Paper w29153, National Bureau of Economic Research, Cambridge, MA.

Maddi, A. and Gingras, Y. (2021). Gender diversity in research teams and citation impact in Economics and Management. Journal of Economic Surveys, 35(5):1381-1404.

Meade, E. E., Starr, M., and Bansak, C. (2021). Changes in Women's Representation in Economics: New Data from the AEA Papers and Proceedings. FEDS Notes, Board of Governors of the Federal Reserve System, Washington.

Newman, M. E. J. (2003). Mixing patterns in networks. Physical Review E, 67(2):026126.

Sarsons, H. (2017). Recognition for Group Work: Gender Differences in Academia. American Economic Review, 107(5):141-145.

Sarsons, H., Gërxhani, K., Reuben, E., and Schram, A. (2021). Gender Differences in Recognition for Group Work. Journal of Political Economy, 129(1):101-147.

Tang, C., Ross, K., Saxena, N., and Chen, R. (2011). What's in a Name: A Study of Names, Gender Inference, and Gender Behavior in Facebook. In Xu, J., Yu, G., Zhou, S., and Unland, R., editors, Database Systems for Advanced Applications, volume 6637, pages 344-356, Berlin, Heidelberg. Springer Berlin Heidelberg. 


\section{A Supplementary information}

Table A.1: NBER working papers by program and decade

\begin{tabular}{lrrrrrr}
\hline Program & $1970 s$ & $1980 s$ & $1990 s$ & $2000 s$ & $2010 s$ & Overall \\
\hline Labor Studies & 40 & 459 & 651 & 870 & 1,081 & 3,101 \\
Economic Fluctuations and Growth & 28 & 465 & 491 & 921 & 1,083 & 2,988 \\
Public Economics & 40 & 447 & 564 & 828 & 993 & 2,873 \\
International Finance and Macroeconomics & 14 & 378 & 469 & 732 & 662 & 2,255 \\
International Trade and Investment & 14 & 374 & 518 & 631 & 525 & 2,062 \\
Monetary Economics & 14 & 430 & 340 & 389 & 514 & 1,687 \\
Asset Pricing & 0 & 0 & 232 & 610 & 627 & 1,470 \\
Productivity, Innovation, and Entrepreneurship & 8 & 100 & 234 & 371 & 563 & 1,276 \\
Corporate Finance & 1 & 1 & 132 & 436 & 560 & 1,130 \\
Health Economics & 11 & 82 & 115 & 354 & 497 & 1,060 \\
Development of the American Economy & 2 & 55 & 180 & 328 & 379 & 944 \\
Industrial Organization & 0 & 0 & 82 & 300 & 432 & 814 \\
Economics of Aging & 1 & 33 & 126 & 237 & 340 & 737 \\
Health Care & 0 & 0 & 100 & 248 & 316 & 665 \\
Environment and Energy Economics & 0 & 1 & 6 & 138 & 483 & 628 \\
Economics of Education & 0 & 0 & 1 & 209 & 411 & 621 \\
Children & 1 & 2 & 35 & 247 & 297 & 582 \\
Political Economics & 0 & 0 & 0 & 141 & 415 & 556 \\
Law and Economics & 6 & 20 & 57 & 188 & 231 & 501 \\
Development Economics & 0 & 0 & 0 & 0 & 462 & 462 \\
Technical Working Papers & 0 & 38 & 96 & 112 & 95 & 342 \\
None & 215 & 24 & 60 & 7 & 0 & 306 \\
\hline Total & 396 & 2,908 & 4,489 & 8,296 & 10,970 & 27,059 \\
\hline
\end{tabular}

Note: Papers associated with $n$ programs contribute $1 / n$ papers to each program's count, which I round to the nearest integer. 


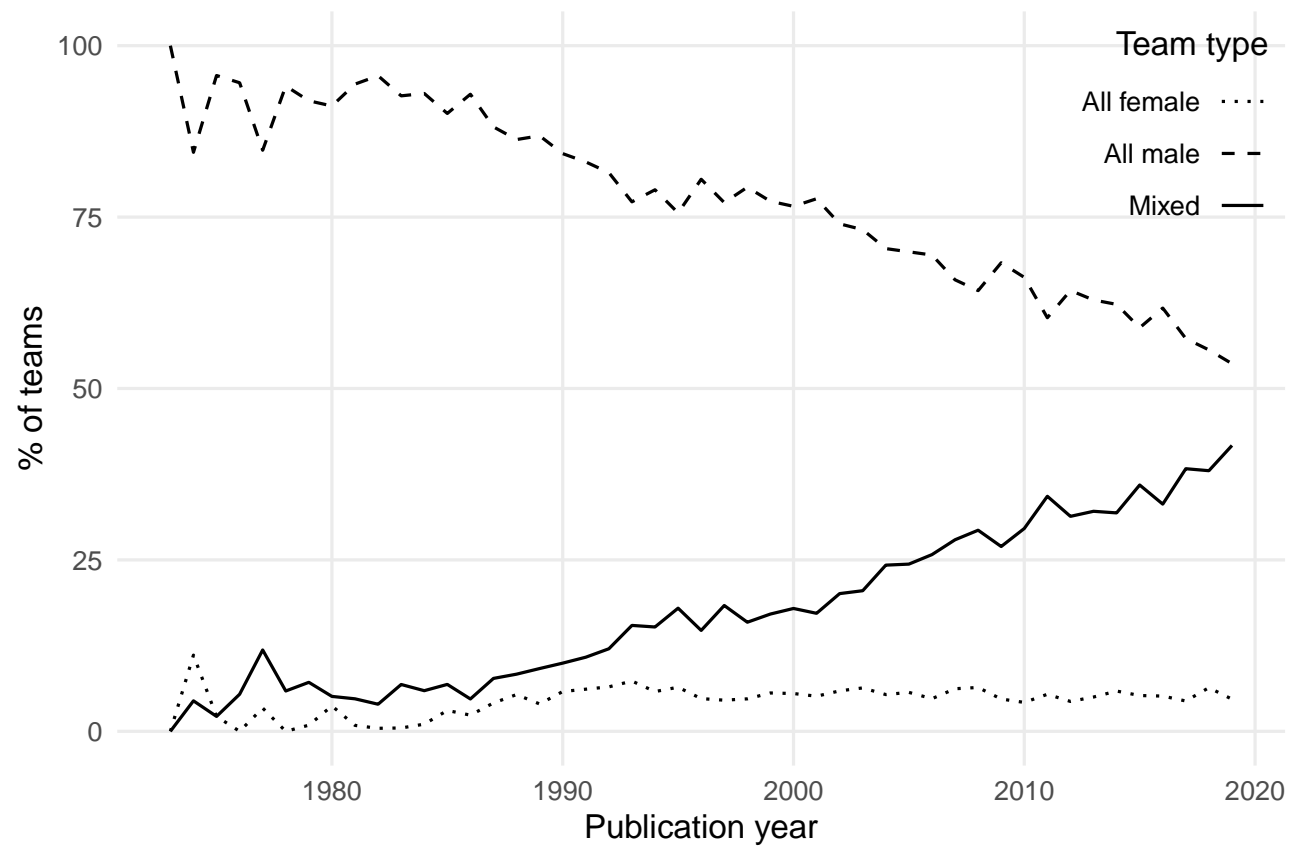

Figure A.1: Shifting co-authorship team compositions on NBER working papers

Notes: Vertical axis measures percentage of papers published each year with each co-authorship team type. Percentages exclude teams with at least one author with unknown gender. "Mixed" teams have at least one male and one female. 


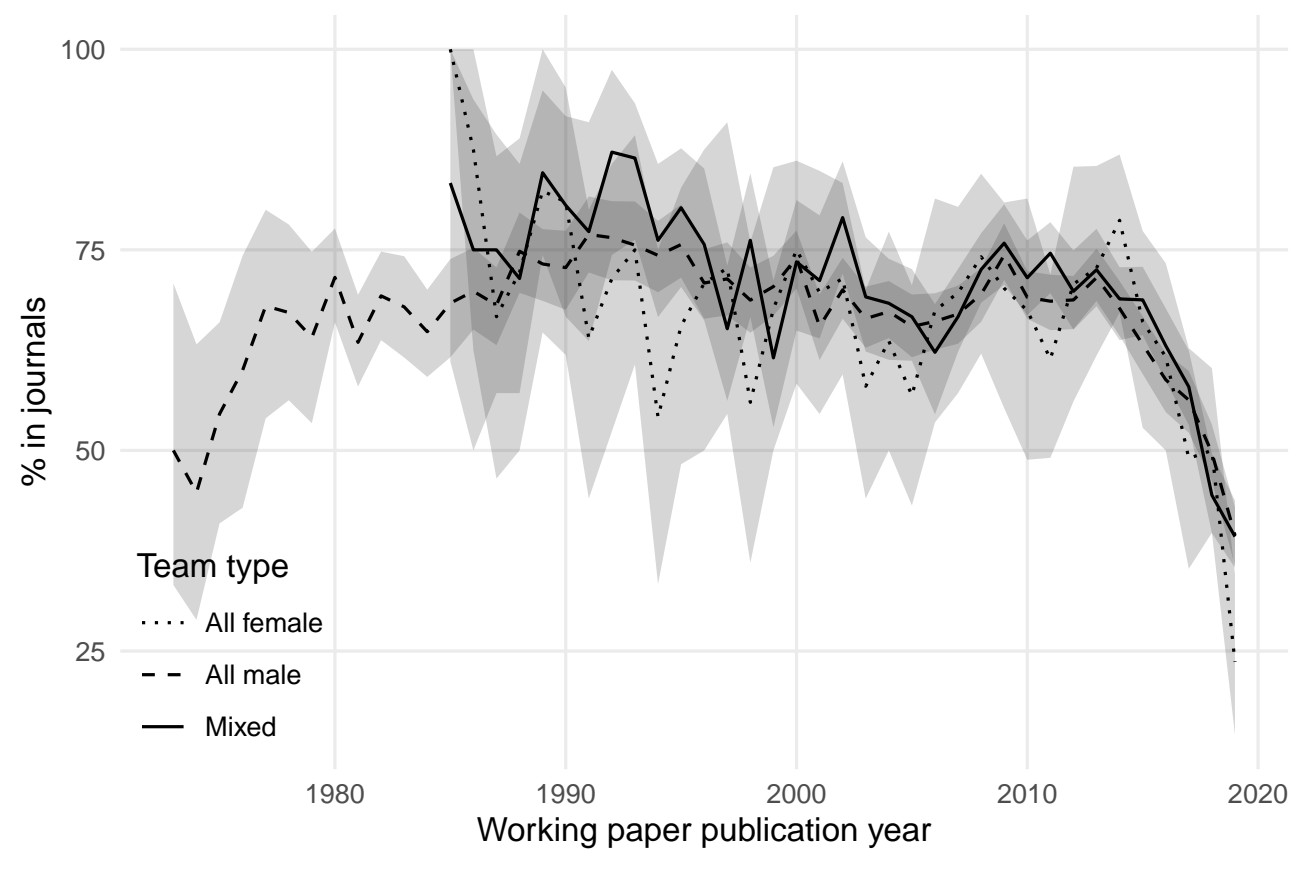

Figure A.2: Journal appearances of NBER working papers

Notes: Vertical axis measures percentage of papers published each year, by each co-authorship team type, that subsequently appeared (or will appear) in peer-reviewed journals as of March 2022. "Mixed" teams have at least one male and one female. Shaded regions represent bootstrapped $95 \%$ confidence intervals around means; darker regions represent interval overlaps. Intervals around each observation (i.e., type-year pair) constructed by computing percentage in each of 200 bootstrap resamples of the underlying data, then computing $2.5 \%$ and $97.5 \%$ quantiles of distribution of bootstrapped percentages. Pre-1985 observations in "All female" and "Mixed" series omitted for visual clarity-there were few such teams in that period, making journal appearance rates noisy and uninformative. Decline in all series in recent years likely reflects papers still undergoing peer-review. 


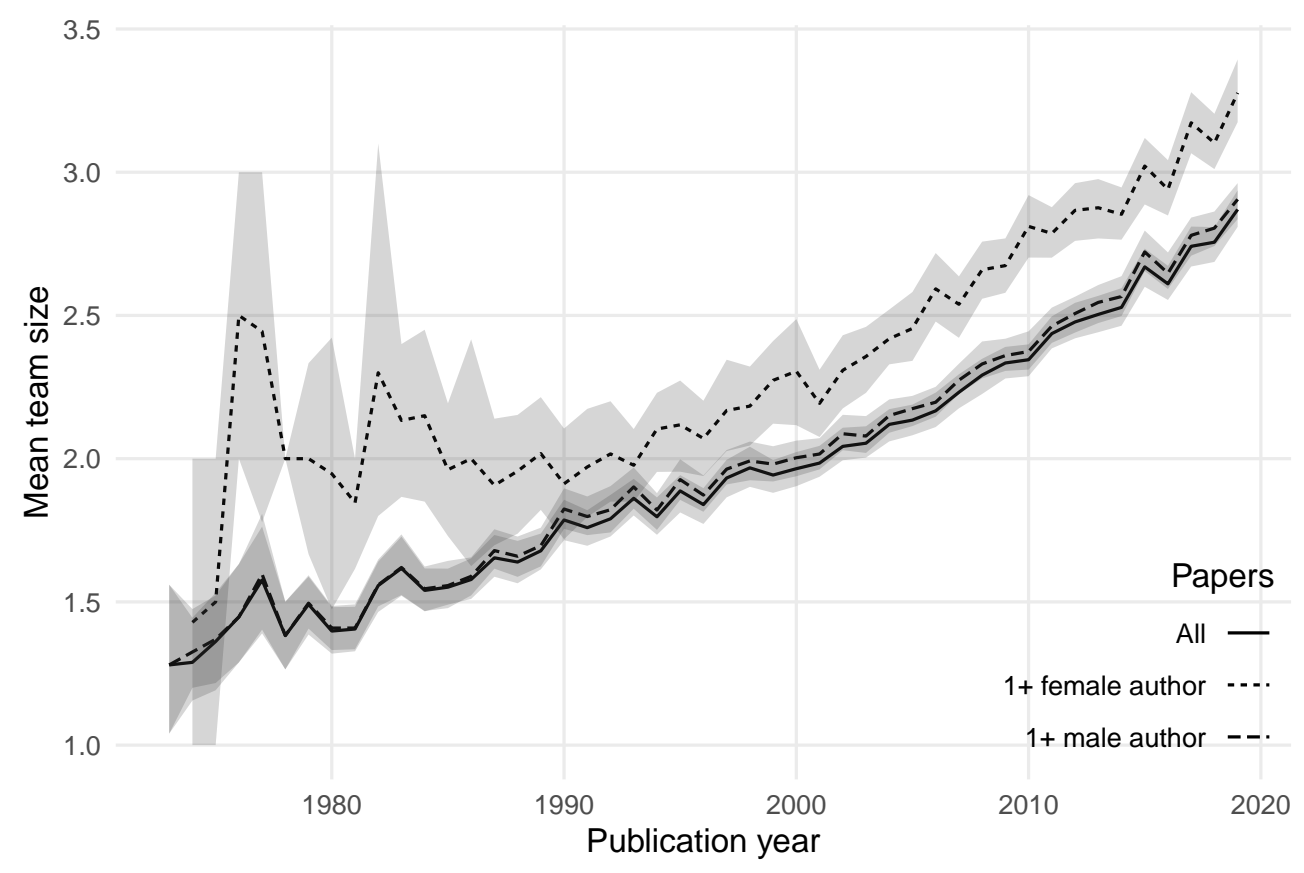

Figure A.3: Bootstrapped 95\% confidence intervals around means displayed in Figure 1

Notes: See Figure 1 notes for series definitions. Intervals around each observation (i.e., series-year pair) constructed by computing mean team size in each of 200 bootstrap resamples (with replacement) of team size distributions, then computing $2.5 \%$ and $97.5 \%$ quantiles of distribution of bootstrapped means. Intervals shown as shaded regions; darker regions represent interval overlaps. Intervals for "All" and " $1+$ male author" series overlap significantly because most teams contained at least one man. 


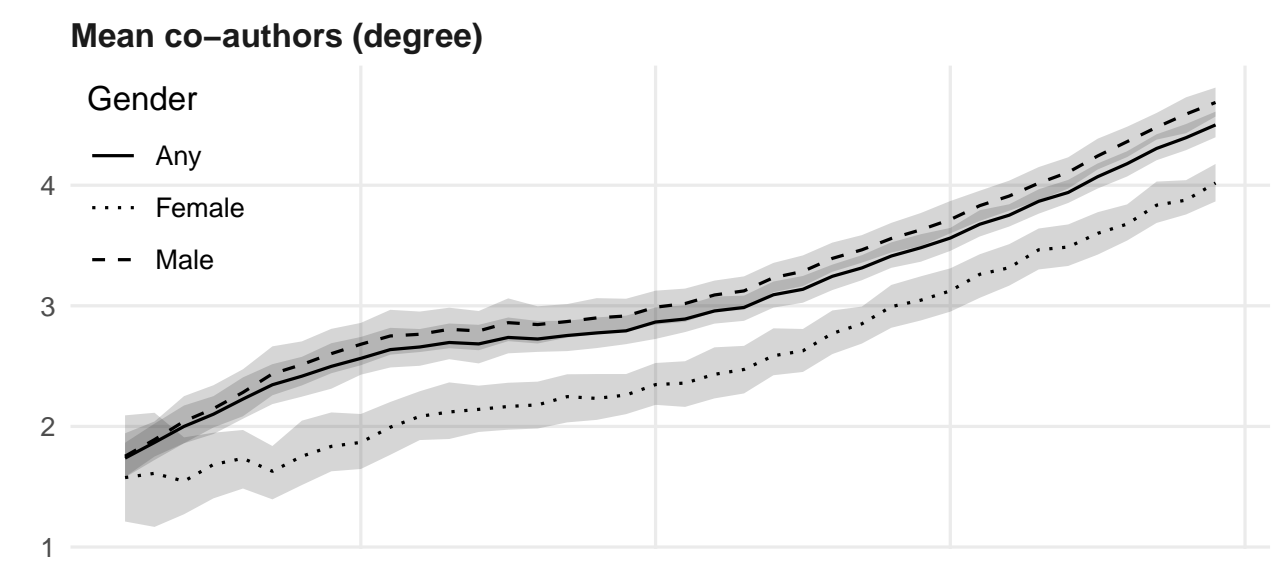

Mean co-authored papers

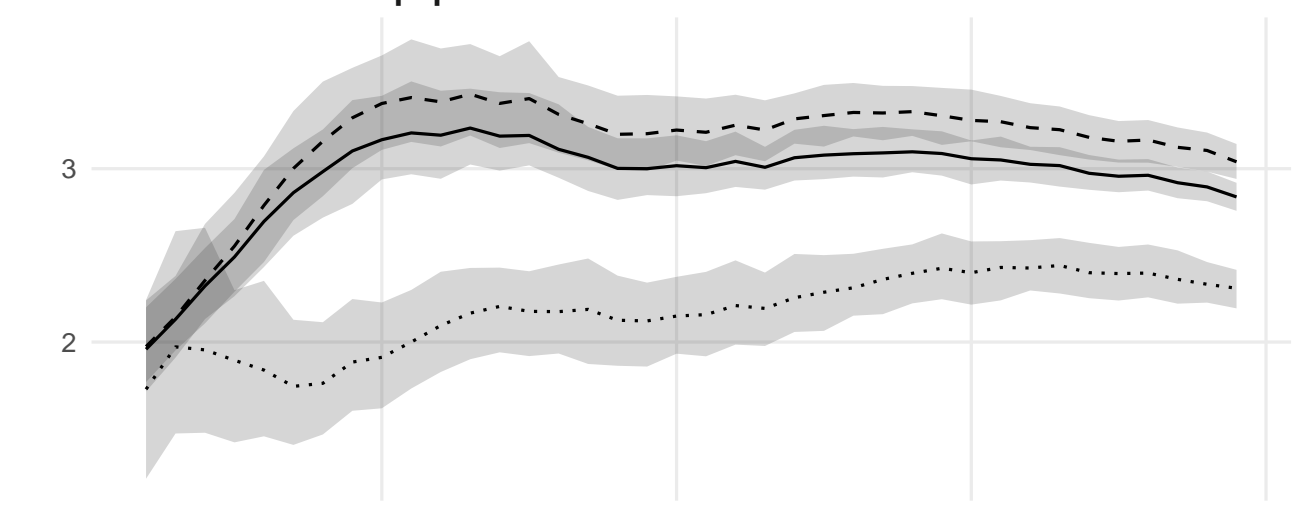

Mean repeat co-authors

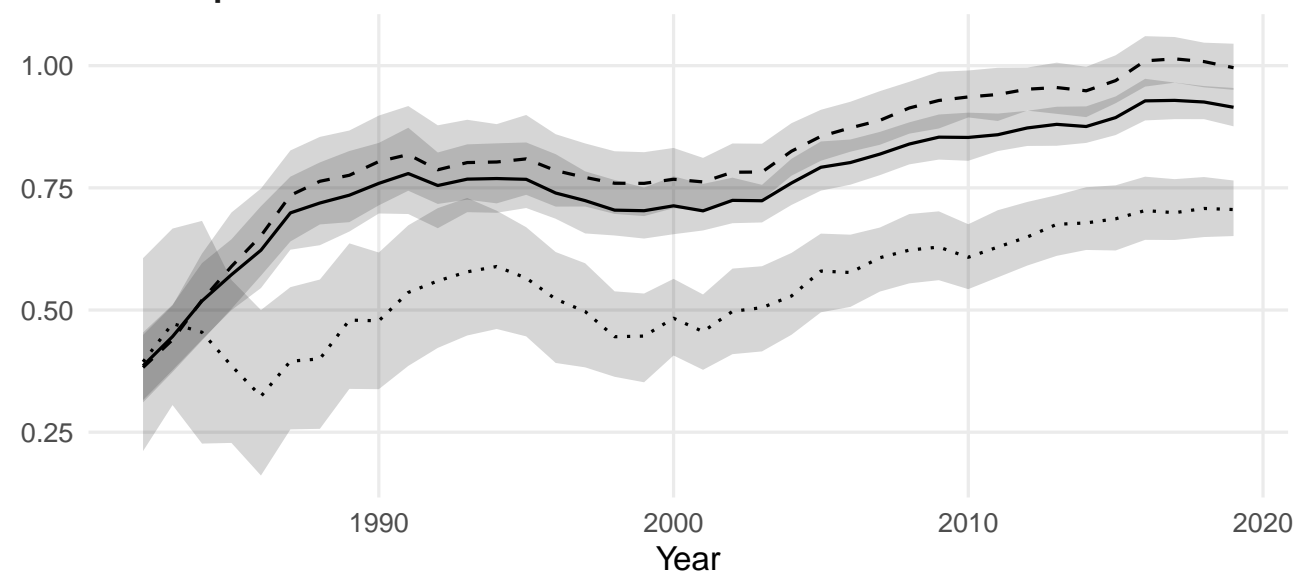

Figure A.4: Bootstrapped 95\% confidence intervals around means displayed in Figure 2

Notes: See Figure 2 notes for series definitions. Intervals around each observation (i.e., series-gender-year tuple) constructed by computing mean in each of 200 bootstrap resamples (with replacement) of the underlying data, then computing $2.5 \%$ and $97.5 \%$ quantiles of distribution of bootstrapped means. Intervals shown as shaded regions; darker regions represent interval overlaps. 